\title{
Early complications of permanent pacemaker implantation: no difference between dual and single chamber systems
}

\author{
Rajesh K Aggarwal, Derek T Connelly, Simon G Ray, Jeff Ball, Richard G Charles
}

\begin{abstract}
Objective-To evaluate the incidence of intraoperative and early postoperative complications (up to two months after implant) of endocardial permanent pacemaker insertion in all patients undergoing a first implant at a referral centre. Methods-Prospective evaluation of all endocardial pacemaker implantation procedures performed from April 1992 to January 1994 carried out by completion of standard audit form at implant. Patients' demographic data, medical history, details of pacemaker hardware used, and any complications were noted. Follow up information was also collected prospectively onto standard forms at pacemaker outpatient clinic.

Setting-United Kingdom tertiary referral cardiothoracic centre.

Patients -1088 consecutive patients underwent implantation of their first endocardial.permanent pacemaker from April 1992 to January 1994. Implant and follow up data were available for 1059 (97.3\%) patients at analysis. The median (range) age was 77 years (16-99); $51 \cdot 2 \%$ were male.
\end{abstract}

Results-Dual chamber units were implanted in $54.1 \%$ of patients, single chamber atrial in $5.2 \%$, and ventricular in $40.7 \%$. A temporary pacing lead was present at implant in $22.9 \%$ of patients. Most $(93.6 \%)$ implants were performed via the subclavian vein. Immediate complications were rare: eight $(0 \cdot 8 \%)$ patients developed pneumothorax requiring medical treatment and 11 (1.0\%) an insignificant pneumothorax. There was no significant difference in the pneumothorax rate for dual chamber (DDD) compared with single chamber systems. Arterial puncture without sequelae was documented in $2.7 \%$ of attempts at subclavian vein cannulation. A total of 35 patients (3.3\%) required reoperation; the reoperation rate for dual chamber $(3.5 \%)$ was similar to that for single chamber (3.1\%) systems. Electrode displacement $(n=15,1.4 \%)$ was the most common reason for reoperation. Atrial lead displacement $(n=10$, $1.6 \%$ of atrial leads) was significantly more common than ventricular lead dis- placement ( $n=5,0.5 \%$ of ventricular leads, $P=0 \cdot 047)$. There was no difference in electrode displacement rates for dual $(1 \cdot 6 \%)$ compared with single $(1 \cdot 2 \%)$ chamber systems. Pacemaker pocket infection led to reoperation in 10 patients (six dual, four single chamber, $P=$ not significant) and was significantly more common in patients who had a temporary pacing lead in place at implant $(2.9 \%)$ than in those who did not $(0.4 \%, P$ $=0.0014)$. Five patients $(0.5 \%)$ required reoperation for generator erosion (two dual, three single chamber, $P=$ not significant) and a further five for drainage of haematoma or a serous fluid collection (three dual, two single chamber, $P=$ not significant). Complications that did not require reoperation were also rare. Undersensing occurred in 10 patients $(0.9 \%)$. Atrial undersensing $(n=8)$ was significantly more common than ventricular undersensing ( $n=2, P=0.017)$. All patients were successfully treated by reprogramming of sensitivity. Superficial wound infection was treated successfully with antibiotics in nine patients (six dual, three single chamber, $P=$ not significant). Three patients with DDD generators developed sustained atrial fibrillation: two required reprogramming to VVI mode and one required cardioversion.

Conclusions-Permanent pacing in a large tertiary referral centre with experienced operators carries a low risk. Infection rates are low, $<1 \%$ overall but significantly higher in patients who undergo temporary pacing before implantation. Lead displacement and undersensing are more likely to occur with atrial than ventricular leads. The overall complication rate for dual chamber pacing, however, is no higher than for single chamber pacing.

(Br Heart f 1995;73:571-575)

Keywords: complications of permanent pacing, dual chamber pacing, single chamber pacing

Permanent pacemaker technology and pacing techniques have advanced considerably since 
their inception in the late 1950 s and permanent pacing is now accepted as being highly cost-effective, safe, and relatively simple to perform. Recently published national guidelines for pacemaker prescription strongly recommend the use of physiological pacemakers. ${ }^{1}$ The use of increasingly sophisticated pacing systems has generated considerable debate, with some authors arguing against dual chamber pacing on the basis of cost, a more complex implantation procedure, and an apparently higher complication rate than single chamber systems. This study was undertaken to assess the complication rate of permanent pacing in a large centre where national pacing guidelines are utilised in the majority (over $85 \%$ ) of endocardial pacemaker implants performed.

\section{Patients and methods}

All patients undergoing their first endocardial pacemaker implant at our institution, a large tertiary referral centre in north west England serving a population of 2.8 million, were entered into the study. We prospectively collected information on 1088 patients who had their first permanent pacemaker implanted from April 1992 to. January 1994. At the time of implant a standard audit form was completed by the operating physician and cardiac technician recording patient demographic data, pacing hardware used, operator and operating time, route of implant, presence of a temporary pacing lead, and use of prophylactic antibiotics. Follow up information was also collected prospectively at the time of outpatient attendance. Particular attention was paid to the occurrence of any complications at implant and follow up. All data were entered into a database set up using commercially available software on a standard IBM compatible computer.

\section{OPERATORS}

Eight operators, each undertaking at least 100 pacing related cases (new implants, generator changes, or system revisions) per annum, implanted almost $92 \%$ of the pacemakers. Three of these operators were inexperienced (on their first 100 cases) during part of the study. Approximately $8 \%$ of implants were undertaken by visiting fellows in training (all inexperienced). Inexperienced operators performed $351(33.1 \%)$ implants in total.

\section{STATISTICAL ANALYSIS}

Differences in proportions were compared using the $\chi^{2}$ test with Yates' correction where appropriate. Continuously variable data were analysed using the Mann-Whitney $U$ test. Significance was defined as $\mathbf{P}<0.05$.

\section{Results}

PATIENT CHARACTERISTICS

A total of 1088 consecutive patients had their first permanent endocardial pacemaker implanted at our centre between April 1992 and January 1994. Implant and follow up data (up to two months after implantation) were available for $1059(97 \cdot 3 \%)$ of these at the time of analysis. Some 542 patients $(51.2 \%)$ were male. The mean (SD) age at implant was $74.8(12 \cdot 2)$ years and median (range) age 77 (16-99) years.

\section{PACEMAKERS AND OPERATORS}

Dual chamber units were implanted in $54 \cdot 1 \%$ of patients, single chamber atrial in $5.2 \%$, and ventricular in $40.7 \%$. Of the 351 pacemakers implanted by inexperienced operators, $50.4 \%$ were dual chamber compared with $55.9 \%$ of units implanted by experienced operators ( $P=$ not significant) (table 1). Mean (SD) implant times were significantly longer for dual chamber systems $(49 \cdot 8$ (18.3) $\mathrm{min}$ ) than single chamber systems (35.4 (16.4) $\mathrm{min}, P<0.0001)$. The subclavian vein route was employed in $93.6 \%$ of all implants. Of the 68 pacemakers implanted by cephalic cut down, only 23 (34\%) were dual chamber compared with $550(5.5 \%)$ of 991 units implanted by the subclavian route. Mean (SD) operation times were significantly shorter for subclavian implantation $(42 \cdot 8$ $(18.8) \mathrm{min})$ than cephalic implantation $(49 \cdot 6$ (19.7) $\min , \mathrm{P}<0.01$ ).

A temporary pacing lead was present at the time of permanent pacemaker implantation in 242 patients $(22 \cdot 9 \%)$. Prophylactic antibiotics, used at the discretion of the operating physician, were administered preoperatively in 117 patients $(11.0 \%)$. Use of antibiotics was significantly more common in patients who had a temporary pacing lead at implant (74 $(30.6 \%)$ of 242 patients) than in those who did not $(43(5.3 \%)$ of 817 patients, $\mathrm{P}<$ $0.0001)$. Only $27(1.6 \%)$ of 1632 pacing leads used were active fixation (screw in) leads.

\section{INTRAOPERATIVE COMPLICATIONS}

The most common intraoperative complication was inadvertent arterial puncture which occurred in 27 patients $(2.7 \%$ of subclavian insertions): no serious sequelae ensued. Pneumothorax required active medical treatment in eight patients $(0.8 \%)$; five had an intercostal chest drain inserted and three were treated by aspiration. A further 11 patients $(1 \cdot 0 \%)$ developed an insignificant pneumothorax $(<10 \%$ of pulmonary field in chest $x$ ray film with no symptoms or progression in subsequent chest radiograph). This represents an overall rate of $1.9 \%$ of subclavian insertions. There was no significant difference in the pneumothorax rate between

Table 1 Type of pacemaker implanted: comparison by operator experience

\begin{tabular}{lll}
\hline & \multicolumn{2}{l}{ Operator } \\
\cline { 2 - 3 } Pacemaker type & Inexperienced (\%) & Experienced (\%) \\
\hline DDD & $177(50 \cdot 4)$ & $396(55 \cdot 9)$ \\
AAI & $17(4 \cdot 8)$ & $38(5 \cdot 4)$ \\
VVI & $157(44 \cdot 7)$ & $274(38 \cdot 7)$ \\
\hline \multirow{2}{*}{ Inexperienced operators (<100 previous implants) } & per-
\end{tabular}
Inexperienced operators 
Table 2 Complications requiring reoperation: comparison of dual and single chamber devices

\begin{tabular}{lll}
\hline Complication & $\begin{array}{l}\text { Dual } \\
\text { chamber }(\%)\end{array}$ & $\begin{array}{l}\text { Single } \\
\text { chamber (\%) }\end{array}$ \\
\hline Pacemaker pocket infection $(\mathrm{n}=10,0.9 \%)$ & $6(1 \cdot 0)$ & $4(0 \cdot 8)$ \\
Generator erosion $(\mathrm{n}=5,0.5 \%)$ & $2(0.3)$ & $3(0.6)$ \\
Haematoma or serous fluid collection $(\mathrm{n}=5,0.5 \%)$ & $3(0.5)$ & $2(0.4)$ \\
Electrode displacement $(\mathrm{n}=15,1 \cdot 4 \%)$ & $9(1 \cdot 6)$ & $6(1 \cdot 2)$ \\
$\quad$ Atrial $(\mathrm{n}=10,1 \cdot 6 \%$ of atrial leads) & & \\
\hline Ventricular $(\mathrm{n}=5,0.5 \%$ of ventricular leads) & & \\
\hline
\end{tabular}

*Atrial leads were significantly more likely to displace than ventricular leads $(P=0.047)$. dual chamber $(\mathrm{n}=12,2 \cdot 1 \%)$ and single chamber $(n=7,1 \cdot 4 \%)$ devices.

\section{COMPLICATIONS REQUIRING REOPERATION}

A total of 35 patients $(3.3 \%)$ required reoperation within the first two months after implant. Patients with dual chamber systems were no more likely to require reoperation (n $=20,3.5 \%)$ than those with single chamber devices $(n=15,3 \cdot 1 \%)$, ( $P=$ not significant $)$. Reoperation was performed most frequently for electrode displacement ( $n=15$ patients, $1 \cdot 4 \%)$. There was no significant difference in the incidence of reoperation for electrode displacement in patients with dual compared with single chamber pacemakers (table 2). However, reoperation was significantly more often required for atrial ( $n=10,1.6 \%$ of atrial leads) than ventricular ( $n=5,0.5 \%$ of ventricular leads) electrode displacement.

Pacemaker pocket infection required generator and electrode removal with simultaneous (or subsequent) implant of a new system on the contralateral side in 10 patients $(0.9 \%)$. The incidence of infection was not significantly different between those with dual chamber devices $(n=6,1.0 \%)$ and those with single chamber devices $(n=4,0.8 \%)$ (table 2). Pacing system removal for infection was, however, significantly more common in patients who had a temporary pacing lead in situ at the time of permanent pacemaker implantation (seven $(2.9 \%)$ of 242 ) than in those who did not (three $(0.4 \%)$ of $817, \mathrm{P}=$ $0.0014)$. Antibiotic prophylaxis did not significantly affect the reoperation rate for infection. Three $(2 \cdot 6 \%)$ of 117 patients given prophylactic antibiotic treatment developed pacemaker pocket infection compared with seven $(0.7 \%)$ of 942 patients who received no antibiotics ( $\mathrm{P}=$ not significant).

Pacemaker generator erosion in the absence of clinical or microbiological evidence of infection led to reoperation in five patients $(0.5 \%)$. The incidence of generator erosion was not significantly different for dual chamber $(\mathrm{n}=2,0.3 \%)$ compared with single chamber $(n=3,0.6 \%)$ systems. Haematoma or serous fluid collection also required operative drainage in five patients $(0.5 \%)$; the incidence of this complication was also similar for dual $(\mathrm{n}=3,0.5 \%)$ and single $(\mathrm{n}=2$, $0.4 \%$ ) chamber pacemakers (table 2 ).

Inexperienced operators had a non-significantly higher incidence of each of the four complications that led to reoperation (table 3).
Table 3 Complications requiring reoperation: analysis by operator experience

\begin{tabular}{|c|c|c|c|}
\hline \multirow[b]{2}{*}{ Complication } & \multicolumn{2}{|l|}{ Operator } & \multirow[b]{2}{*}{$P$ value } \\
\hline & $\begin{array}{l}\text { Inexperienced } \\
(\%)^{\star}\end{array}$ & $\begin{array}{l}\text { Experienced } \\
(\%)\end{array}$ & \\
\hline \multirow{5}{*}{$\begin{array}{l}\text { Infection }(n=10, \\
0.9 \%) \\
\text { Erosion }(n=5,0.5 \%) \\
\text { Haematoma }(n=5, \\
0.5 \%) \\
\text { Lead displacement } \\
(n=15,1.4 \%) \\
\text { Any complication }\end{array}$} & $5(1 \cdot 4)$ & $5(0.7)$ & NS \\
\hline & $3(0 \cdot 8)$ & $2(0 \cdot 3)$ & NS \\
\hline & $4(1 \cdot 1)$ & $1(0 \cdot 1)$ & NS \\
\hline & $8(2 \cdot 3)$ & $7(1 \cdot 0)$ & NS \\
\hline & $20(5 \cdot 7 \%)$ & $15(2 \cdot 1)$ & 0.0039 \\
\hline
\end{tabular}

^Inexperienced operator ( $<100$ previous implants). NS, not significant.

Table 4 Complications managed without reoperation: comparison of dual and single chamber devices

\begin{tabular}{lll}
\hline Complication & $\begin{array}{l}\text { Dual } \\
\text { chamber (\%) }\end{array}$ & $\begin{array}{l}\text { Single } \\
\text { chamber (\%) }\end{array}$ \\
\hline $\begin{array}{l}\text { Superficial wound infection } \\
\text { (n=9,0.8\%) }\end{array}$ & $6(1 \cdot 0)$ & $3(0 \cdot 6)$ \\
$\begin{array}{l}\text { Undersensing }(\mathrm{n}=10,0.9 \%) \\
\text { Atrial }(\mathrm{n}=8,1 \cdot 3 \% \text { of atrial leads) }\end{array}$ & $4(0 \cdot 8)$ \\
Ventricular $(\mathrm{n}=2,0 \cdot 2 \%$ of ventricular leads) & \\
\hline
\end{tabular}

^Atrial undersensing was significantly more common than ventricular undersensing $(P=0.017)$

The overall incidence of reoperation, however, was significantly greater for patients paced by inexperienced operators ( 20 of 351 , $5.7 \%$ ) compared to those paced by experienced operators $(15$ of $708,2 \cdot 1 \%, P=$ 0.0039).

\section{COMPLICATIONS MANAGED WITHOUT} REOPERATION

There were also a small number of complications that did not require reoperation. $\mathbf{P}$ or $\mathbf{R}$ wave undersensing was the most common of these and occurred in 10 patients $(0.9 \%)$ (table 4). Dual $(n=6,1 \cdot 0 \%)$ and single $(n=$ $4,0.8 \%$ ) chamber pacemakers were almost equally affected. Atrial undersensing $(n=8)$, however, was significantly more common than ventricular undersensing $(n=2, P=$ $0 \cdot 017$ ) (table 4). All patients were successfully treated by reprogramming of sensitivity. Superficial wound infection was reported in nine patients $(0.8 \%)$, six with dual chamber and three with single chamber pacemakers $(P$ = not significant) (table 4). All were treated successfully with a single course of antibiotics. Three patients with DDD generators developed sustained atrial fibrillation; two of these required reprogramming of their generators to VVI mode and one was cardioverted electrically. There was no mortality attributable to pacemaker implantation in this series.

\section{Discussion}

National guidelines for pacemaker prescription, published in $1991^{1}$ encourage the use of physiological pacing systems and have led to much debate about the cost-effectiveness of such systems. ${ }^{2-4}$ In the debate about physiological pacing some authors have pointed to 
the greater difficulty in implanting and following up dual chamber systems ${ }^{3}$ and the apparently higher complication rate for dual than single chamber pacing.

\section{OPERATOR EXPERIENCE}

Studies comparing the complication rates of dual versus single chamber pacemaker implantation have previously reported either no difference 5 or a higher complication rate for dual than single chamber pacing. ${ }^{6}$ In neither of these series, however, were any single chamber atrial pacemakers implanted and in both $<25 \%$ of the implanted units were dual chamber. ${ }^{56}$

Our study was undertaken to assess the complication rate in a large tertiary referral centre in the United Kingdom where over 600 new pacemaker implants are performed annually, the majority ( $>85 \%$ ) in accordance with national guidelines. Although most procedures are performed by cardiologists in the training grades (registrars and senior registrars), all such operators at our centre would be considered "frequent implanters" according to criteria used by Parsonnet et al (performing $>12$ procedures per annum) in their analysis of factors contributing to pacemaker implantation complications. Indeed, over $90 \%$ of the procedures in our series were performed by operators undertaking at least 100 pacemaker related cases per annum and two thirds by experienced operators ( $>100$ previous cases).

Frequent implanters, experienced in dual and single chamber (atrial and ventricular) pacemaker insertion, account for low overall complication rates. Chauhan et $a l^{6}$ failed to acknowledge this fact in a recently published study reporting a higher early complication rate for dual than single chamber pacemaker implantation. The authors analysed early complications after implantation of 2019 pacemakers $(85 \cdot 8 \% \mathrm{VVI}, 14 \cdot 2 \% \mathrm{DDD}$, and no AAI units) by 23 operators over a 6 year period. Fifteen operators in their series were classed as "frequent implanters" and even if these 15 operators had performed all 286 dual chamber implants during the study, the DDD implant rate would be $<3.2$ units/ operator/year. Thus all operators were, in fact, infrequent implanters of DDD systems but frequent implanters of VVI systems. This fact alone may account for the higher complication rate for dual than single chamber pacing reported by Chauhan et al. ${ }^{6}$

\section{VENOUS ACCESS}

The subclavian vein approach, using the introducer method, remains the most widely employed route for pacemaker implantation. ${ }^{8}$ Although Parsonnet et $a l^{7}$ found that this approach contributed significantly to pacemaker implantation complication rates, they were unable to demonstrate a causal relation between complications and the route of access. Although cephalic cut down has been advocated as the route of choice, ${ }^{7}$ most pacemakers in our series were implanted by the subclavian approach (at the operator's discre- tion). The cephalic vein route is relatively unfavourable for introducing two leads and cephalic vein dissection significantly prolongs operation time. The rate of serious pneumothorax in our centre is low $(<1 \%)$. Our low overall complication rate, attributable to experienced high volume operators, is unlikely to be further improved by more frequent use of the cephalic approach.

\section{REPEAT OPERATION}

In our series, the most common reason for reoperation within the first two months after implant was electrode displacement. The overall rate of electrode displacement was $1.4 \%$ and essentially similar for single and dual chamber pacemakers. Atrial leads, however, were over three times as likely to displace as ventricular leads. Our rates of electrode displacement compare favourably with $1.7 \%$ for atrial and ventricular leads reported by Miller et $a l^{9}$ and $0.8 \%$ for ventricular leads only reported by Hess et al. ${ }^{10}$ Although Mueller et $a l^{5}$ reported no significant difference in atrial $(1.3 \%)$ compared with ventricular $(0.9 \%)$ lead displacement, their series of 337 procedures ( $77 \%$ VVI) compared tined ventricular leads with active fixation (screw in) atrial leads. In contrast only $11(1.7 \%)$ of 628 atrial leads and 16 $(1.6 \%)$ of 1004 ventricular leads used in our series were active fixation leads.

Pacemaker pocket infection in our series was uncommon with an incidence of reoperation within 2 months of implant of $0.9 \%$. This is at the lower end of reported rates of $1-7 \%^{11}$; however, our true incidence of infection leading to reoperation may be marginally higher as some patients with pocket infection present late. ${ }^{12}$ We did not routinely use prophylactic antibiotics in this series. These were administered at the discretion of the operating cardiologist and were used more than five times as frequently in patients who had a temporary pacing lead in place at the time of permanent pacemaker insertion than in those who did not. There is no clear evidence to support the routine use of prophylactic antibiotics for pacemaker implantation. In a large randomised trial involving 431 patients, Muers $e t a l^{13}$ found an apparent benefit with seven of nine pocket infections occurring in patients randomised to receive no antibiotics. Their pocket infection rate was $2 \cdot 1 \%$ and the overall reoperation rate $21 \% .{ }^{13}$ More recently, Mounsey et $a l^{14}$ reported a reduction in reoperation rate for infection, with 12 of 13 pocket infections occurring in patients not receiving antibiotic prophylaxis; however, they found no benefit in the subgroup of patients with the highest risk of infection (those with temporary pacing electrodes). The exclusion of $19 \%$ of patients with temporary electrodes from their study may thus have biased the overall results. In addition, their infection rate in patients not treated with antibiotics ( 12 of $368,3.3 \%)$ was significantly higher than ours $(0 \cdot 7 \%)$.

In contrast to the two series reporting a benefit from antibiotic prophylaxis, Bluhm et 
$a l^{15}$ reported no benefit in a small randomised series of 106 patients undergoing pacemaker implantation. In addition, a large (500 patients) prospective randomised study performed at our own institution showed that, with meticulous preoperative skin preparation, use of a topical antibiotic spray into the pacemaker pocket, and close postoperative follow up, patients gained no advantage from routine prescription of prophylactic antibiotics. ${ }^{16}$ Irrespective of the use of antibiotics, patients in our series who had a temporary pacing lead in place at the time of permanent pacemaker implantation were more than seven times as likely to require generator and electrode explant for infection than patients who were not temporarily paced before permanent pacemaker insertion. There seemed to be no obvious relation with duration of temporary pacing, though the absolute number of infected generators was too small to draw a valid conclusion in this regard.

Other problems requiring reoperation were rare and again there was no difference between dual and single chamber pacemakers. Haematoma or serous fluid collection occurred in less than $0.5 \%$ of patients. Generator erosion without infection had a similar incidence within two months of implant. Erosion rates may be marginally higher at prolonged follow up, although this has not been our experience previously in patients followed for up to 1 year. ${ }^{16}$

In conclusion, permanent pacing in a large tertiary referral cardiac centre with operators experienced in single and dual chamber pacemaker implantation carries a low intraoperative and early postoperative risk. Infection rates are less than $1 \%$ overall and there are no differences between dual and single chamber devices. Pacemaker pocket infection, however, is significantly more likely to occur in patients who have a temporary pacing lead in place at the time of permanent pacemaker implantation. Thus, temporary pacing before implantation should be avoided whenever possible. In addition, patients with temporary pacing leads and unequivocal indication for permanent pacing should undergo permanent implantation at the earliest available opportunity. Although electrode displacement and undersensing occur more frequently with atrial than ventricular leads, dual chamber systems confer no higher risk of these events than do single chamber systems. Indeed, the overall complication rate for dual chamber pacing is no higher than for single chamber pacing.

We gratefully acknowledge the invaluable assistance of all the Liverpool Cardiothoracic Centre cardiac technicians with collection of implant and follow up data. We thank Dr N Waterfield, Dr G J Green (Ysbyty Glan Clwyd, Bodelwyddan), Mrs G M Gaddie, and $\operatorname{Dr} R T$ Maxwell (Ysbyty Gwynedd, Bangor) for assistance with collection of follow up data.

1 Clarke M, Sutton R, Ward D, et al. Recommendations for pacemaker prescription for symptomatic bradycardia. pacemaker prescription for symptomatic bradycardia. Report of a working party of the British Pacing and
Electrophysiology Group. Br Heart f 1991;66:185-91.

2 de Belder MA, Linker NJ, Jones S, Camm AJ, Ward DE. Cost implications of the British Pacing and Electrophysiology Group's recommendations for pacing. $B M F$ 1992;305:861-5.

3 Petch MC. Who needs dual chamber pacing? $B M F$ 1993;307:215-6.

4 Channon KM, Cripps T, Ormerod O. Recommendations for pacing [letter]. BM尹 1992;305:1431-2.

5 Mueller X, Sadeghi H, Kappenberger L. Complications after single versus dual chamber pacemaker implantation. PACE 1990;13:711-4.

6 Chauhan A, Grace AA, Newell SA, et al. Early complications after dual chamber versus single chamber pacemaker implantation. PACE 1994;17(Pt II):2012-5.

7 Parsonnet V, Bernstein AD, Lindsay B. Pacemakerimplantation complication rates: an analysis of some implantation complication rates: an analysis of some contributing

8 Bernstein $\mathrm{AD}$, Parsonnet V. Survey of cardiac pacing in the United States in 1989. Am f Cardiol 1992;69:331-8.

9 Miller GB, Leman PB, Kratz JM, et al. Comparison of lead dislodgement and pocket infection rates after pacemaker implantation in the operating room versus the catheterisation laboratory. Am Heart $\mathcal{f} 1980 ; 115: 1048$.

10 Hess DS, Gertz WE, Morady F, et al. Permanent pacemaker implantation in the cardiac catheterisation laboratory: the subclavian vein approach. Cathet Cardiovasc Diagn 1982;8:453.

11 Phibbs B, Marriott HJL. Complications of permanent transvenous pacing. $N$ Engl f Med 1985;22:1428-32.

12 Wohl B, Peters RW, Carliner N, et al. Late unheralded pacemaker pocket infection due to Staphylococcus epidermidis: a new clinical entity. PACE 1982;5:190.

13 Muers MF, Arnold AG, Sleight P. Prophylactic antibiotics for cardiac pacemaker implantation. A prospecbiotics for cardiac pacemaker implan.

14 Mounsey JP, Griffith MJ, Tynan M, et al. Antibiotic prophylaxis in permanent pacemaker implantation: a prospective randomised trial. $\mathrm{Br}$ Heart $\mathcal{f}$ 1994;72: $339-43$.

15 Bluhm G, Nordlander R, Ransjo U. Antibiotic prophylaxis in pacemaker surgery: a prospective double blind trial with systemic administration of antibiotic versus placebo at implantation of cardiac pacemakers. $P A C E$ 1986;9(5):720-6.

16 Ramsdale DR, Charles RG, Rowlands DB, Singh SS, Gautam PC, Faragher EB. Antibiotic prophylaxis for pacemaker implantation: a prospective randomised trial. PACE 1984;7:844-9. 